\title{
De novo food allergy in pediatric liver transplantation recipients
}

\author{
Ratchaneewan Sinitkul, ${ }^{1}$ Wiparat Manuyakorn, ${ }^{1}$ Wasu Kamchaisatian, ${ }^{1}$ Soamarat Vilaiyuk, ${ }^{1}$ Suwat Benjaponpitak,${ }^{1}$ \\ Chatmanee Lertudompholwanit, ${ }^{2}$ Suporn Treepongkaruna ${ }^{2}$
}

\begin{abstract}
Background: Food allergy (FA) prevalence is increasing in pediatric liver transplantation (LT). However, the clinical course is still limited.

Objective: This retrospective cohort study aimed to identify the prevalence, risk factors, and the natural history of de novo FA in children post LT.

Methods: Medical records of pediatric LT recipients from Jan 2001 - Dec 2014 were reviewed. De novo FA was diagnosed by symptoms after exposure to culprit food occurring after LT, and improvement after diet elimination. FA was confirmed if reproduced symptoms after re-challenge or documented sensitization or indicated gastrointestinal eosinophilia.

Results: Among 46 post LT children, 54.3\% developed de novo FA at a median time of 12.2 months [Interquartile range (IQR) 6.2, 21.3 months] post LT. The confirmed FA was 39.1\%. Gastrointestinal symptom was the most common manifestation followed by skin, anaphylaxis, and others. Culprit foods were cow's milk, shellfish, egg, wheat, soybean, peanut, coconut, fish and monosodium glutamate. The risk factors of FA were transplantation during age below 2 years [hazard ratio (HR), 2.62; 95\% confidence interval (CI), $1.04-6.59 ; p=0.03)$, atopic history in family $(\mathrm{HR}, 5.67 ; 95 \% \mathrm{CI}$, 1.33 - 24.12; $p=0.01$ ), and Epstein-Barr (EBV) viremia (HR, 2.39; 95\% CI, $1.02-5.63 ; p=0.04)$.
\end{abstract}

Conclusions: de novo FA in pediatric LT is not uncommon. Age at LT younger than 2 years, family history of atopy, and EBV viremia are associated with developing FA. Development of tolerance after elimination culprit diets for 3 years is similar to general population.

Key words: Food allergy, liver transplantation, tolerance, outgrown, de novo food allergy

\section{From:}

${ }^{1}$ Division of Allergy and Immunology

${ }^{2}$ Division of Gastroenterology

Department of Pediatrics, Faculty of Medicine Ramathibodi Hospital, Mahidol University, Bangkok, Thailand

\section{Introduction}

Food allergy (FA) is defined as an immune-mediated adverse reaction to food, divided by immunopathology to IgE-mediated, non IgE-mediated, and mixed type. ${ }^{1}$ Over the past 2 decades, the prevalence of FA was doubled and its phenotypic expression increased in Westernized societies. ${ }^{2}$ In the US $28 \%$ of 480 children from birth cohort study reported adverse reactions to foods, but in only $8 \%$ of these reactions were reproduced. ${ }^{3}$ In Thailand, the prevalence of adverse food reactions by questionnaire in school children under age of 7 years ranged from $6.25 \%$ to $13.1 \%$ and the

\section{Corresponding author:}

Suporn Treepongkaruna

Department of Pediatrics, Faculty of Medicine Ramathibodi Hospital,

Mahidol University

270 Rama VI Road, Ratchathewi, Bangkok, Thailand 10400

E-mail: suporntr@gmail.com, suporn.tre@mahidol.ac.th

prevalence of confirmed IgE-mediated FA by food challenge were $0.45 \%$ to $1.11 \% .{ }^{4,5}$ However, the prevalence of FA are highlighted in some special conditions particularly the patients undergo solid organ transplantation. ${ }^{6}$

Presently, liver transplant (LT) is the treatment of choice in liver failure and provides an excellent outcome and survival rate. $^{7}$ The quality of life and long term health aspects are increasingly concerned. Interestingly, the incidence of novel FA or de novo FA following LT has been reported to be 5 to $57 \%$ in various populations but the pathogenesis remains 
elucidate. ${ }^{6,8-11}$ While the incidence of this condition was increasing, several risk factors such as tacrolimus use, EBV viremia and younger age at transplantation have been reported but these remain inconclusive. ${ }^{8,9,12,13}$ Furthermore, the natural history is also unclear leading to the reluctance of health care providers and child caregivers for dietary management. Therefore, this study aimed to 1) determine the prevalence of de novo FA, 2) identify risk factors of de novo FA, and 3) demonstrate the natural history of de novo FA in post LT children.

\section{Methods \\ Study design}

Retrospective cohort study

\section{Patients and Samples}

Patients who underwent LT between Jan 2001 and Dec 2014 were reviewed using patient's records and telephone interviewing by one researcher. The patients who had survived after LT at least 30 months were included in this study. Demographic data including gender, underlying diseases, indication for LT, age at LT, follow-up period, immunosuppressive drug regimen, and atopic history such as allergic rhinitis (AR), asthma, atopic dermatitis (AD) and FA were collected. The FA history focusing on the onset, symptoms, treatment, and reaction after re-consumption either by incidence or intention were gathered. The evidence of sensitization was identified by skin prick test and specific $\operatorname{IgE}(\mathrm{s} \operatorname{IgE})$. The patients were routinely followed-up by the gastroenterologists (ST and CL). Patients with suspected FA were evaluated and diagnosed by the allergists (RS and WM). De novo FA was defined as symptoms and/or signs of FA developed after LT. We classified FA into 2 groups which were confirmed and probable FA as the following criteria; $;^{14,15}$

Confirmed FA was defined as the symptoms and/or signs of FA that resolved after food elimination, combined with either reproducible symptoms and/or signs when reintroducing the culprit foods or the evidence of food sensitization (SPT or sIgE) or evidence of gastrointestinal eosinophilia without other specific causes.

Probable FA was diagnosed from a history of symptoms and/or signs of FA that improved after avoidance without the re-challenge of culprit foods, nor laboratory tests to confirm the food reactions. The other causes that mimic FA such as infection, drug adverse effects were excluded.

IgE-mediated FA included urticaria, angioedema, acute onset gastrointestinal or respiratory symptoms, and anaphylaxis. The reaction occurred within 2 hours after ingestion of the culprit foods. ${ }^{14}$ The evidence of food sensitization was used to support the IgE-mediated reaction.

Non IgE-mediated FA included subacute or delayed onset of gastrointestinal symptoms ( $>2$ hours after taking the culprit foods). They were food protein-induced proctocolitis, food protein-induced enterocolitis, and food protein-induced enteropathy. ${ }^{14}$
Mixed IgE- and non-IgE mediated FA included moderate to severe $\mathrm{AD}$ and eosinophilic gastrointestinal disorders (EGIDs). In order to diagnose EGIDs, esophagogastroduodenoscopy and endoscopic biopsies were performed and the diagnosis was based on the abnormal numbers or distribution of eosinophils in tissue histopathology. ${ }^{15}$

Evidence of food sensitization was determined by SPT or prick to prick (PTP) or sIgE. In SPT and PTP, histamine chloride and sodium chloride $(0.9 \%)$ were used as positive and negative controls, respectively. Skin test applicator of Duotip-test (Lincoln Diagnostics, Illinois, USA) was performed on the skin at forearm for either the SPT or PTP. The standard food allergenic extracts (ALK Abelló, Port Washington, NY, USA) of cow's milk, soy, wheat, egg yolk, egg white, and mixed shellfish were applied for SPT. The selected cooked and/or fresh foods were performed for prick-to-prick skin test (PTP) in cases of unavailable commercial standard allergen. The sIgE was measured by the ImmunoCAP assay (Thermo Fisher Scientific, Uppsala, Sweden). The wheal size $>3 \mathrm{~mm}$ larger than negative control in SPT and PTP or sIgE $>0.35 \mathrm{IU} / \mathrm{mL}$ were considered the positive result.

Outgrowing of FA was defined as the ability to re-consume the culprit foods without any reaction for more than 1 month.

Study protocol was approved by the Research Ethical Committee of the Faculty of Medicine Ramathibodi Hospital. Informed consent was obtained from a caregiver before enrollment in the study.

\section{Statistical analysis}

Data were analyzed using the statistics package SPSS 17.0 and Stata software version 12.0. A descriptive analysis was performed on all study variables, using median with quartile for numeric variables. The differences of baseline characteristic were assessed by Chi-square and Mann-Whitney $U$ test. Risk factors for de novo FA post LT were determined by the univariate analysis of log-rank test and presented as Hazard Ratio. Probability of having de novo FA post LT was described by Kaplan-Meier survival analysis. The differences with a $P$ value less than 0.05 are considered statistically significant. The Log-rank test comparing two survival rates was calculated to determine the statistical power.

\section{Results \\ Patient demographics}

Between January 1, 2001 and Dec 30, 2014, a total of 52 pediatric patients underwent LT at Ramathibodi Hospital. Forty-six patients survived and were eligible for including in the study. Twenty-five patients (54.3\%) had de novo FA and 18 of them $(39.1 \%)$ had confirmed FA (Figure 1). The demographic data are described in Table 1 that the baseline characteristics were not different between de novo FA and non de novo FA except family history of atopy $(p=0.031)$. There were eight donors who reported the symptoms of allergic rhinitis and only one of shellfish allergy. Standard immunosuppression regimen was composed of tacrolimus starting within 24 hours 


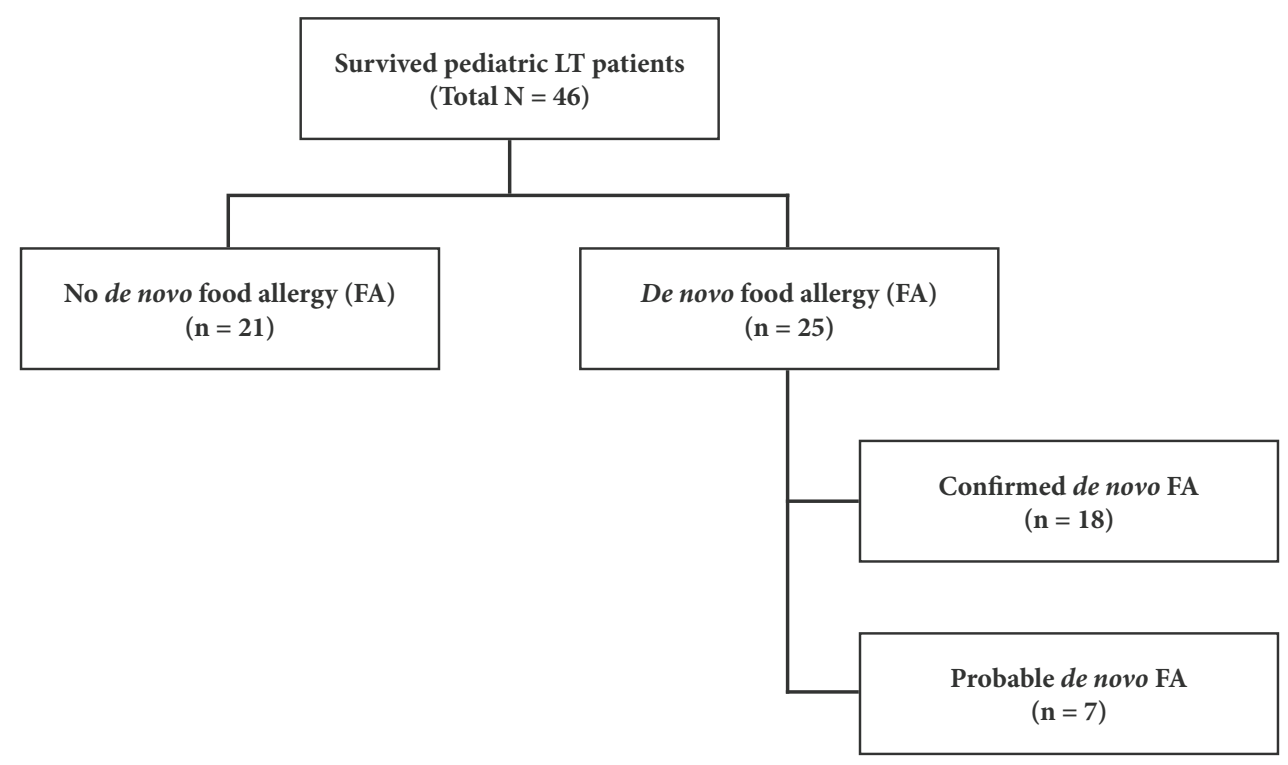

Figure 1. The overall de novo food allergy (FA) outcome in survived pediatric liver transplantation recipients

Table 1. Demographics of the patients

\begin{tabular}{|c|c|c|c|c|}
\hline \multirow[b]{2}{*}{ Variable } & \multirow{2}{*}{$\begin{array}{c}\text { Overall } \\
\text { n (\%) }\end{array}$} & \multicolumn{2}{|c|}{ De novo food allergy } & \multirow[b]{2}{*}{$P$ value $\$$} \\
\hline & & $\begin{array}{c}\text { De novo FA } \\
\text { n (\%) }\end{array}$ & $\begin{array}{c}\text { Non de novo FA } \\
\mathrm{n}(\%)\end{array}$ & \\
\hline Gender (Male) & $16(34.8)$ & $8(32.0)$ & $8(38.1)$ & $0.665^{\mathrm{a}}$ \\
\hline Median age at transplantation; months (IQR) & $\begin{array}{c}19.1 \\
(15.3,34.2)\end{array}$ & $\begin{array}{c}16.7 \\
(12.2,23.3)\end{array}$ & $\begin{array}{c}29.2 \\
(17.7,64.4)\end{array}$ & $0.107^{\mathrm{b}}$ \\
\hline Median follow-up period; months (IQR) & $\begin{array}{c}59.5 \\
(57.2,92.8)\end{array}$ & $\begin{array}{c}67.4 \\
(50.2,98.6)\end{array}$ & $\begin{array}{c}55.8 \\
(42.8,95.7)\end{array}$ & $0.337^{\mathrm{b}}$ \\
\hline Median age at study; months (IQR) & $\begin{array}{c}89.3 \\
(65.9,128.9)\end{array}$ & $\begin{array}{c}87.1 \\
(60.7,121.7)\end{array}$ & $\begin{array}{c}103.8 \\
(66.8,173.4)\end{array}$ & $0.225^{\mathrm{b}}$ \\
\hline $\begin{array}{l}\text { Indication for transplantation } \\
\text { Biliary atresia } \\
\text { Others }^{\mathrm{d}}\end{array}$ & $\begin{array}{c}37(80.4) \\
9(19.6)\end{array}$ & $\begin{array}{c}22(88.0) \\
3(12.0)\end{array}$ & $\begin{array}{c}15(71.4) \\
6(28.6)\end{array}$ & $0.264^{\mathrm{c}}$ \\
\hline PELDs (IQR) & $\begin{array}{c}18.0 \\
(15,21)\end{array}$ & $\begin{array}{c}18.0 \\
(14.5,21.0)\end{array}$ & $\begin{array}{c}18.5 \\
(14.3,21.8)\end{array}$ & $0.660^{\mathrm{b}}$ \\
\hline Prior atopy before LT & $5(10.9)$ & $3(12.0)$ & $2(9.5)$ & $0.788^{\mathrm{a}}$ \\
\hline First-degree family history of atopy & $16(34.8)$ & $12(48.0)$ & $4(19.0)$ & $0.031^{\mathrm{a}, *}$ \\
\hline $\begin{array}{l}\text { History of atopy in donor } \\
\text { Allergic rhinitis } \\
\text { Food allergy }\end{array}$ & $\begin{array}{c}8(17.4) \\
8 \\
1\end{array}$ & $\begin{array}{c}6(24.0) \\
6 \\
1\end{array}$ & $\begin{array}{c}2(9.5) \\
2 \\
0\end{array}$ & $0.260^{c}$ \\
\hline $\begin{array}{l}\text { Long term immunosuppression } \\
\text { Tacrolimus } \\
\text { Tacrolimus and MMF }\end{array}$ & $\begin{array}{l}26(56.5) \\
20(43.5)\end{array}$ & $\begin{array}{c}16(64.0) \\
9(32.0)\end{array}$ & $\begin{array}{l}10(47.6) \\
11(52.4)\end{array}$ & $0.282^{\mathrm{a}}$ \\
\hline
\end{tabular}

Abbreviation; FA, food allergy; IQR, interquartile range; LT, liver transplant; MMF, mycophenolate mofetyl; PELDs, Pediatric End-stage Liver Disease score;

${ }^{\text {a }}$ Chi-square test

${ }^{\mathrm{b}}$ Mann Whitney U test

${ }^{\mathrm{c}}$ Fisher's exact test

after the operation and continuing for life-long, combined with corticosteroid in the first 6 months after LT. Additional mycophenolate mofetil (MMF) was given to some patients as clinical indicated. d Allagille syndrome, progressive familial intrahepatic cholestasis (PFIC) type 2, tyrosinemia, glycogen storage disease, hemangioendothelioma ${ }^{*} P<0.05$

${ }^{s}$ The difference between de novo FA and non de novo FA

\section{Clinical manifestations, allergic food and types of FA}

Twenty-two out of 25 food allergic patients (88\%) had multiple food allergies. The median time to diagnosis de novo FA after LT was 12.2 months (IQR 6.2, 21.3 months). EGIDs were diagnosed in 4 patients. 
Table 2. The numbers of culprit food and outgrowing rate of each food allergen

\begin{tabular}{|lcc|}
\hline Culprit food & $\begin{array}{c}\text { De novo FA } \\
\text { n/n total (\%) }\end{array}$ & $\begin{array}{r}\text { Outgrowing } \\
\text { n/n total (\%) }\end{array}$ \\
\hline Cow's milk & $18 / 85(21.2)$ & $12 / 18(66.7)$ \\
\hline Soy & $14 / 85(16.5)$ & $4 / 14(28.5)$ \\
\hline Shellfish & $14 / 85(16.5)$ & $8 / 14(57.1)$ \\
\hline Hen's egg & $13 / 85(15.3)$ & $6 / 13(46.2)$ \\
\hline Fish & $8 / 85(9.5)$ & $1 / 8(12.5)$ \\
\hline Wheat & $6 / 85(7.0)$ & $4 / 6(66.7)$ \\
\hline Others & $12 / 85(14.1)$ & $0 / 12(0)$ \\
\hline
\end{tabular}

Table 3. Clinical features of de novo FA in pediatric LT
Among 85 items of allergic food items in 25 de novo FA patients, IgE-mediated FA was the most common reaction (49.4\%), follow by mixed (29.4\%), and non IgE-mediated reaction $(21.2 \%)$. The major culprit foods were milk, soy, shellfish, hen's egg and others (Table 2).

The presenting symptoms were gastrointestinal symptoms (52\%), followed by skin (urticaria/angioedema, eczema), anaphylaxis, anemia and respiratory (stridor) as described in Table 3. One case of multiple food allergy post LT has identical twins and his twin was healthy with no clinical of any atopic diseases.

\section{Risk factors for de novo FA post LT}

Risk factors for de novo FA post LT were the age at LT of less than 2 years [hazard ratio (HR), 2.62; 95\% confidence interval (CI), $1.04-6.59 ; p=0.03)$, Epstein-Barr $(\mathrm{EBV})$ viremia of more than 100 copies prior to developing FA (HR, 2.39; 95\% CI,

\begin{tabular}{|c|c|c|c|c|c|c|c|c|c|}
\hline No. & Indication & Outgrowing & $\begin{array}{l}\text { Age at LT } \\
\text { (months) }\end{array}$ & Culprit food & $\begin{array}{c}\text { sIgE } \\
(\mathrm{IU} / \mathrm{mL})\end{array}$ & $\begin{array}{l}\text { SPT/PTP } \\
\text { [wheal } \\
\text { diameter } \\
(\mathrm{mm})]\end{array}$ & Clinical & $\begin{array}{l}\text { Type of } \\
\text { allergic } \\
\text { reaction }\end{array}$ & $\begin{array}{l}\text { Food } \\
\text { allergy }\end{array}$ \\
\hline 1 & BA & 21.6 & 36 & $\begin{array}{l}\text { CM } \\
\text { Soy } \\
\text { Shellfish } \\
\text { EW }\end{array}$ & $\begin{array}{l}3.19 \\
0.36 \\
1.25 \\
1.52\end{array}$ & $\begin{array}{l}\text { NA } \\
\text { NA } \\
\text { NA } \\
\text { NA }\end{array}$ & $\mathrm{AD}$ & Mixed & $\begin{array}{l}\text { Confirmed } \\
(\mathrm{OC})\end{array}$ \\
\hline 2 & BA & 19.2 & 51.6 & CM, shellfish & NA & NA & $\begin{array}{l}\text { Diarrhea } \\
\text { (EGID) }\end{array}$ & Mixed & Probable \\
\hline 3 & $\begin{array}{l}\text { Tyrosinemia } \\
\text { type } 1\end{array}$ & 15.6 & 15.6 & $\begin{array}{l}\text { Shrimp } \\
\text { Fish (Nile tilapia) } \\
\text { Blood cockle }\end{array}$ & $\begin{array}{l}\text { NA } \\
\text { NA } \\
\text { NA }\end{array}$ & $\begin{array}{c}3 \\
10 \text { (cooked) } \\
\text { NA }\end{array}$ & angioedema & $\begin{array}{l}\text { IgE- } \\
\text { mediated }\end{array}$ & $\begin{array}{l}\text { Confirmed } \\
\text { (OC) }\end{array}$ \\
\hline 4 & BA & 22.8 & 9.6 & $\begin{array}{l}\text { Shrimp } \\
\text { Cashew nut }\end{array}$ & $\begin{array}{l}\mathrm{NA} \\
\mathrm{NA}\end{array}$ & $\begin{array}{l}\text { NA } \\
\text { NA }\end{array}$ & $\begin{array}{l}\text { Urticaria, } \\
\text { angioedema, } \\
\text { anaphylaxis }\end{array}$ & $\begin{array}{l}\text { IgE- } \\
\text { mediated }\end{array}$ & $\begin{array}{l}\text { Confirmed } \\
\text { (accidental } \\
\text { OC) }\end{array}$ \\
\hline 5 & BA & 15.6 & 21.6 & $\begin{array}{l}\text { EW } \\
\text { Wheat } \\
\text { CM } \\
\text { Soy } \\
\text { Shrimp }\end{array}$ & $\begin{array}{l}15.5 \\
29.8 \\
37.6 \\
14.8 \\
6.79\end{array}$ & $\begin{array}{l}\text { NA } \\
\text { NA } \\
\text { NA } \\
\text { NA } \\
\text { NA }\end{array}$ & $\begin{array}{l}\text { Diarrhea } \\
\text { (EGID), } \\
\text { anemia, } \\
\text { hypoalbuminemia }\end{array}$ & Mixed & Confirmed \\
\hline 6 & BA & 36 & 40.8 & $\begin{array}{l}\text { EY } \\
\text { Wheat } \\
\text { Soy } \\
\text { Fish } \\
\text { Shrimp }\end{array}$ & $\begin{array}{c}1.42 \\
0.51 \\
1.57 \\
6.39(\mathrm{Cod}) \\
0.39\end{array}$ & $\begin{array}{l}\text { NA } \\
\text { NA } \\
\text { NA } \\
\text { NA } \\
\text { NA }\end{array}$ & $\begin{array}{l}\text { Diarrhea } \\
\text { (EGID), } \\
\text { anemia }\end{array}$ & Mixed & Confirmed \\
\hline 7 & BA & 20.4 & 30 & $\begin{array}{l}\text { Soy } \\
\text { Shellfish }\end{array}$ & $\begin{array}{l}8.99 \\
3.52\end{array}$ & $\begin{array}{l}\text { NA } \\
\text { NA }\end{array}$ & $\begin{array}{l}\text { Diarrhea, } \\
\text { mouth itching }\end{array}$ & $\begin{array}{l}\text { Mixed, } \\
\text { IgE- } \\
\text { mediated }\end{array}$ & Confirmed \\
\hline 8 & BA & 34.8 & 24 & $\begin{array}{l}\text { CM } \\
\text { Soy } \\
\text { Shellfish }\end{array}$ & $\begin{array}{l}0.01 \\
0.00 \\
\text { NA }\end{array}$ & $\begin{array}{l}\text { NA } \\
\text { NA } \\
\text { NA }\end{array}$ & $\begin{array}{l}\text { AD, Diarrhea } \\
\text { (EGID) }\end{array}$ & Mixed & $\begin{array}{l}\text { Confirmed } \\
\text { (accidental } \\
\text { OC) }\end{array}$ \\
\hline 9 & $\begin{array}{l}\text { Allagille } \\
\text { syndrome }\end{array}$ & 46.8 & 6 & $\mathrm{CM}$ & NA & NA & Diarrhea & $\begin{array}{l}\text { Non-IgE } \\
\text { mediated }\end{array}$ & Probable \\
\hline 10 & BA & 34.8 & 12 & $\begin{array}{l}\text { Fish } \\
\text { (Nile tilapia) } \\
\text { Soy } \\
\text { CM } \\
\text { Shrimp } \\
\text { Egg }\end{array}$ & $\begin{array}{c}\text { NA } \\
5.76 \\
22.4 \\
0.53 \\
6(\mathrm{EW}) \\
9.28(\mathrm{EY})\end{array}$ & $\begin{array}{c}15,10 \\
\text { (fresh, cooked) } \\
\text { NA } \\
\text { NA } \\
\text { NA } \\
\text { NA }\end{array}$ & $\begin{array}{l}\text { Angioedema, } \\
\text { vomiting, } \\
\text { abdominal } \\
\text { pain, rash, } \\
\text { AD }\end{array}$ & $\begin{array}{l}\text { IgE- } \\
\text { mediated }\end{array}$ & Confirmed \\
\hline
\end{tabular}


Table 3. (Continued) Clinical features of de novo FA in pediatric LT

\begin{tabular}{|c|c|c|c|c|c|c|c|c|c|}
\hline No. & Indication & Outgrowing & $\begin{array}{l}\text { Age at LT } \\
\text { (months) }\end{array}$ & Culprit food & $\begin{array}{c}\text { sIgE } \\
(\mathrm{IU} / \mathrm{mL})\end{array}$ & $\begin{array}{l}\text { SPT/PTP } \\
\text { [wheal } \\
\text { diameter } \\
(\mathrm{mm})]\end{array}$ & Clinical & $\begin{array}{l}\text { Type of } \\
\text { allergic } \\
\text { reaction }\end{array}$ & $\begin{array}{l}\text { Food } \\
\text { allergy }\end{array}$ \\
\hline 11 & BA & 10.8 & 12 & $\begin{array}{l}\text { Coconut milk } \\
\text { Peanut } \\
\text { Soy } \\
\text { Shrimp } \\
\text { Squid }\end{array}$ & $\begin{array}{l}\text { NA } \\
48.6 \\
36.2 \\
17.0 \\
\text { NA }\end{array}$ & $\begin{array}{c}13 \\
\text { NA } \\
\text { NA } \\
\text { NA } \\
5\end{array}$ & $\begin{array}{l}\text { Angioedema, } \\
\text { urticaria, } \\
\text { vomiting, } \\
\text { diarrhea }\end{array}$ & $\begin{array}{l}\text { IgE- } \\
\text { mediated }\end{array}$ & Confirmed \\
\hline 12 & BA & 4.8 & 12 & $\begin{array}{l}\text { Shellfish } \\
\text { CM } \\
\text { Egg } \\
\text { Peanut }\end{array}$ & $\begin{array}{c}0.05 \\
0.13 \\
0.03(\mathrm{EW}) \\
0.03(\mathrm{EY}) \\
\text { NA }\end{array}$ & $\begin{array}{l}\text { NA } \\
\text { NA } \\
\text { NA } \\
\text { NA }\end{array}$ & $\begin{array}{l}\text { Angioedema, } \\
\text { diarrhea, AD }\end{array}$ & $\begin{array}{l}\text { IgE- } \\
\text { mediated, } \\
\text { non IgE- } \\
\text { mediated } \\
\text { and mixed }\end{array}$ & $\begin{array}{l}\text { Confirmed } \\
\text { (accidental } \\
\text { OC) }\end{array}$ \\
\hline 13 & BA & 15.6 & 36 & Squid & NA & NA & Angioedema & $\begin{array}{l}\text { IgE- } \\
\text { mediated }\end{array}$ & Probable \\
\hline 14 & $\mathrm{BA}$ & 20.4 & 24 & $\mathrm{MSG}^{\ddagger}$ & NA & NA & Urticaria & $\begin{array}{l}\text { IgE- } \\
\text { mediated }\end{array}$ & $\begin{array}{l}\text { Confirmed } \\
\text { (OC) }\end{array}$ \\
\hline 15 & BA & 14.4 & 6 & $\begin{array}{l}\text { Coconut milk } \\
\text { Pumpkin }{ }^{\ddagger} \\
\text { Fish } \\
\text { CM } \\
\text { Egg } \\
\text { Soy } \\
\text { Shellfish }\end{array}$ & $\begin{array}{c}\text { NA } \\
\text { NA } \\
0.03(\mathrm{Cod}) \\
8.93 \\
23.9(\mathrm{EW}) \\
12.9(\mathrm{EY}) \\
4.68 \\
0.32\end{array}$ & $\begin{array}{l}\text { NA } \\
\text { NA } \\
\text { NA } \\
\text { NA } \\
\text { NA } \\
\\
\text { NA } \\
\text { NA }\end{array}$ & $\begin{array}{l}\text { Angioedema, } \\
\text { diarrhea, } \\
\text { anaphylaxis }\end{array}$ & $\begin{array}{l}\text { IgE- } \\
\text { mediated }\end{array}$ & Confirmed \\
\hline 16 & $\mathrm{ALF}$ & 10.8 & 2.4 & $\begin{array}{l}\text { CM } \\
\text { EW } \\
\text { Coconut milk } \\
\text { Shrimp } \\
\text { Soy }\end{array}$ & $\begin{array}{l}\text { NA } \\
\text { NA } \\
\text { NA } \\
\text { NA } \\
\text { NA }\end{array}$ & $\begin{array}{c}\text { Negative } \\
4 \\
\text { Negative } \\
\text { Negative } \\
\text { Negative }\end{array}$ & $\begin{array}{l}\text { Urticaria, } \\
\text { angioedema, } \\
\text { AD, anemia }\end{array}$ & $\begin{array}{l}\text { IgE- } \\
\text { mediated, } \\
\text { mixed }\end{array}$ & $\begin{array}{l}\text { Confirmed } \\
\text { (accidental } \\
\text { OC) }\end{array}$ \\
\hline 17 & $\mathrm{BA}$ & 33.6 & 12 & $\mathrm{CM}$ & NA & NA & Diarrhea & $\begin{array}{l}\text { Non-IgE- } \\
\text { mediated }\end{array}$ & $\begin{array}{l}\text { Confirmed } \\
\text { (accidental } \\
\text { OC) }\end{array}$ \\
\hline 18 & $\mathrm{BA}$ & 9.6 & 2.4 & $\begin{array}{l}\text { CM } \\
\text { Wheat } \\
\text { Egg } \\
\text { Soy } \\
\text { Peanut } \\
\text { Shrimp } \\
\text { Fish }\end{array}$ & $\begin{array}{c}79.8 \\
>100 \\
84.5(\mathrm{EW}), \\
13.0(\mathrm{EY}) \\
22.4 \\
13.1 \\
41.9 \\
36.7(\mathrm{Cod})\end{array}$ & $\begin{array}{l}\text { NA } \\
\text { NA } \\
\text { NA } \\
\\
\text { NA } \\
\text { NA } \\
\text { NA } \\
\text { NA }\end{array}$ & $\begin{array}{l}\text { AD, stridor } \\
\text { (vocal cord } \\
\text { edema) }\end{array}$ & $\begin{array}{l}\text { IgE- } \\
\text { mediated, } \\
\text { mixed }\end{array}$ & $\begin{array}{l}\text { Confirmed } \\
\text { (accidental } \\
\text { OC) }\end{array}$ \\
\hline 19 & $\mathrm{BA}$ & 9.6 & 9.6 & CM, soy & NA & NA & Diarrhea & Mixed & Probable \\
\hline 20 & $\mathrm{BA}$ & 9.6 & 6 & $\begin{array}{l}\text { CM } \\
\text { Soy } \\
\text { Crab } \\
\text { EW }\end{array}$ & $\begin{array}{l}0.64 \\
3.93 \\
0.03 \\
0.22\end{array}$ & $\begin{array}{l}\text { NA } \\
\text { NA } \\
\text { NA } \\
\text { NA }\end{array}$ & Diarrhea, AD & Mixed & Probable \\
\hline 21 & BA & 13.2 & 3.6 & $\begin{array}{l}\text { CM } \\
\text { Wheat }\end{array}$ & $\begin{array}{c}48.0 \\
>100\end{array}$ & $\begin{array}{l}\text { NA } \\
\text { NA }\end{array}$ & $\mathrm{AD}$ & Mixed & $\begin{array}{l}\text { Confirmed } \\
\text { (accidental } \\
\text { OC) }\end{array}$ \\
\hline 22 & $\mathrm{BA}$ & 15.6 & 2.4 & $\begin{array}{l}\text { Soy } \\
\text { CM } \\
\text { Egg } \\
{ }^{*} \text { CMPA } \\
\text { diagnosed before } \\
\text { LT }\end{array}$ & $\begin{array}{c}0.00 \\
0.01 \\
0.12(\mathrm{EW}) \\
0.12(\mathrm{EY})\end{array}$ & $\begin{array}{l}\text { NA } \\
\text { NA } \\
\text { NA }\end{array}$ & $\begin{array}{l}\text { Anaphylaxis } \\
\text { (soy), AD, } \\
\text { diarrhea }\end{array}$ & $\begin{array}{l}\text { IgE- } \\
\text { mediated, } \\
\text { non IgE- } \\
\text { mediated }\end{array}$ & Probable \\
\hline 23 & BA & 16.8 & 6 & $\begin{array}{l}\text { CM } \\
\text { Soy } \\
\text { Fish (Nile tilapia) } \\
\text { Wheat }\end{array}$ & $\begin{array}{l}0.04 \\
0.01 \\
\text { NA } \\
0.03\end{array}$ & $\begin{array}{c}\text { NA } \\
\text { NA } \\
\text { NA } \\
4\end{array}$ & $\begin{array}{l}\text { Hypersecretion, } \\
\text { vomiting, diarrhea } \\
\text { and fever } \\
\text { suspected FPIES }\end{array}$ & $\begin{array}{l}\text { Mixed, } \\
\text { non IgE- } \\
\text { mediated }\end{array}$ & Probable \\
\hline
\end{tabular}


Table 3. (Continued) Clinical features of de novo FA in pediatric LT

\begin{tabular}{|c|c|c|c|c|c|c|c|c|c|}
\hline No. & Indication & Outgrowing & $\begin{array}{l}\text { Age at LT } \\
\text { (months) }\end{array}$ & Culprit food & $\begin{array}{c}\text { sIgE } \\
(\mathrm{IU} / \mathrm{mL})\end{array}$ & $\begin{array}{c}\text { SPT/PTP } \\
\text { [wheal } \\
\text { diameter } \\
(\mathrm{mm})]\end{array}$ & Clinical & $\begin{array}{l}\text { Type of } \\
\text { allergic } \\
\text { reaction }\end{array}$ & $\begin{array}{l}\text { Food } \\
\text { allergy }\end{array}$ \\
\hline 24 & BA & 21.6 & 14.4 & $\mathrm{CM}$ & 0.03 & NA & $\begin{array}{l}\text { Anemia, } \\
\text { diarrhea }\end{array}$ & $\begin{array}{l}\text { Non IgE- } \\
\text { mediated }\end{array}$ & $\begin{array}{l}\text { Confirmed } \\
\text { (OC) }\end{array}$ \\
\hline 25 & $\mathrm{BA}$ & 20.4 & 3.6 & $\mathrm{CM}$ & NA & NA & Diarrhea & $\begin{array}{l}\text { Non IgE- } \\
\text { mediated }\end{array}$ & $\begin{array}{l}\text { Confirmed } \\
\text { (OC) }\end{array}$ \\
\hline
\end{tabular}

Abbreviation; AD, atopic dermatitis; ALF, Acute liver failure; BA, biliary atresia; CM, cow's milk; CMPA, cow's milk protein allergy; EGID, Eosinophilic gastroenteritis disease; EW, egg white; EY, egg yolk; FPIES: Food Protein-Induced Enterocolitis Syndrome; NA: Not available; OC: oral challenge; PTP: prick to prick; sIgE: serum specific IgE; SPT: skin prick test

${ }^{\ddagger}$ Other culprit foods in this study were MSG, coconut milk, cashew nut, and pumpkin
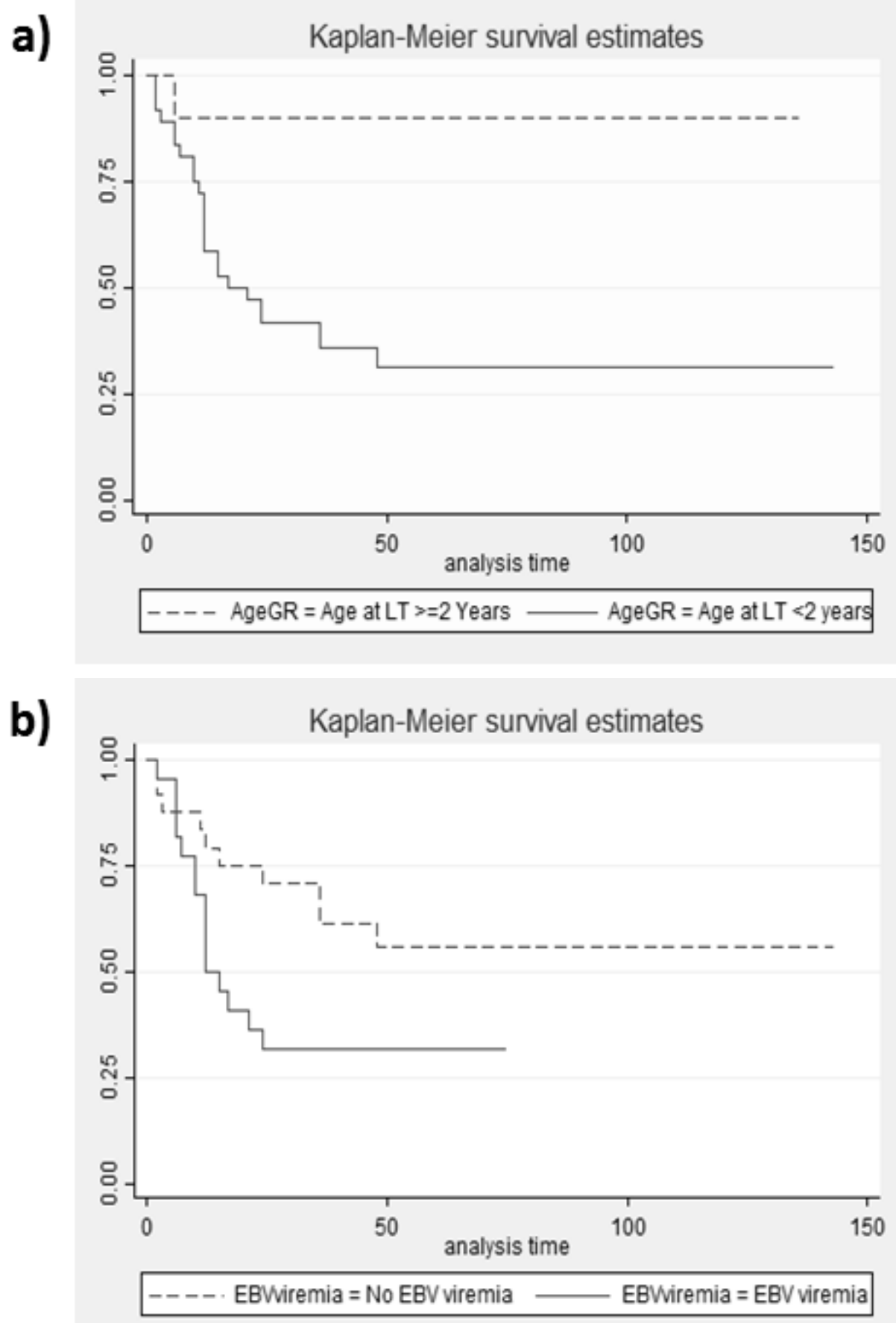

Figure 2. The Kaplan-Meier survival estimates of risk factors for de novo FA post liver transplantation (LT). a) The age of LT at less than 2 years, b) Epstein-Barr (EBV) viremia more than 100 copies prior to FA had developed 


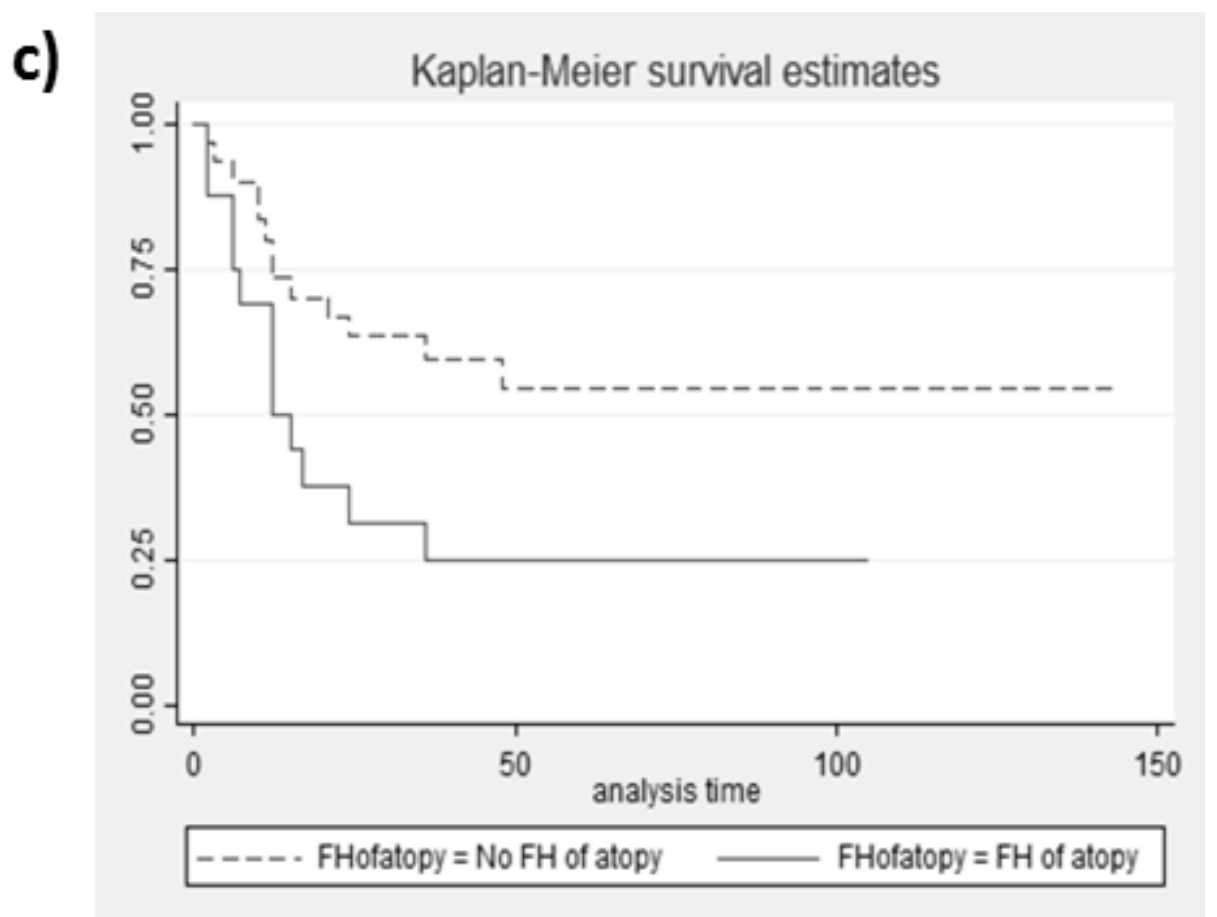

Figure 2. (Continued) c) family history of atopic diseases. The dash line and solid line represent the absence and presence of these factors, respectively.

$1.02-5.63 ; p=0.04)$, and family history of atopic diseases; hay fever, $\mathrm{AD}, \mathrm{FA}, \mathrm{AR}$, and/or asthma (HR, 5.67; 95\% CI, 1.33 - 24.12; $p=0.01$ ) (Figure 2a-c). CMV viremia was found in $68 \%$ of $d e$ novo FA but none in non de novo FA group. The small number of patients in each group led to an insufficient statistical power to provide a conclusive analysis of risk factors for CMV, hepatitis $\mathrm{B}$, and herpes simplex virus infection in this population. Other factors including predisposing individual atopic history before LT, severity of end-stage liver disease that measured by Pediatric End-Stage Liver Disease (PELD) score, are not statistically different. Log-rank test comparing two survival rates of $\mathrm{Ha}=0.76$, Hazards ratio 2.39 (lowest $\mathrm{HR}$ in our study). The statistical power is enough at power of 80 , total $n=48$. The sub-group analysis of the biliary atresia participants were found the similar trend of the risk factors of $\mathrm{FH}$ of atopy (HR 3.12;95\%CI, 1.32-7.36; $\mathrm{p}=0.009$ ) and EBV viremia (HR 1.80; $95 \% \mathrm{CI}, 0.76-4.24 ; \mathrm{p}=0.182)$. However, the factor of age at LT $<$ 2 years was not enough to calculate.

\section{Outgrowing of food allergy}

Among 25 de novo FA patients, 19\% develops tolerance to at least 1 food allergen after 3 years of elimination diet. Due to many patients had multiple FA, the outgrowing rate within 3 years after food elimination was determined by numbers of food items. Of the total 85 items of culprit food, 35 (41.2\%) items were reported of tolerance after re-challenge. The most common outgrown food allergen were cow's milk and wheat occurring in $66.7 \%$, followed by shellfish, egg, soy, and fish (Table 2).

\section{Discussion}

This retrospective study highlighted the high prevalence of de novo FA (54.3\%) in the 46 survived children underwent
LT. It was clearly higher than that of global and Thai children but similar to previous reports of FA in pediatric LT. The prevalence of FA in normal population is approximately $1 \%$ to $10.8 \%$ in westernized countries by food challenges and $3 \%$ to $35 \%$ by self-reported allergy. ${ }^{2}$ In Thailand, the nationwide survey of the FA has not been reported. However, two studies of questionnaire survey and oral food challenge (OFC) in Bangkok (2005) and northern Thailand (2011) have shown the prevalence of FA of $13.1 \%$ (positive OFC 1.1\%) in Thai preschool children and $6.25 \%$ (OFC $0.45 \%$ ) in school children. ${ }^{4,5}$ Our study demonstrated the same prevalence as the several worldwide reports of FA after LT which range from $6 \%$ to 57\%. ${ }^{9}$ The prevalence rate of de novo FA in this study was high compared to other reports, possibly due to the selective criteria of FA that we included all types of food allergic reactions; IgE-, non IgE-, and mixed types.

The pattern of FA presentation, types of reaction, food allergens, and outgrowing rate in LT children were mostly similar to those of normal children population. Our study demonstrated that gastrointestinal and skin manifestations were common presentations, and the most common reaction was IgE-mediated (49.4\%). EGIDs were found in 4 cases or $8.7 \%$ of survived LT which was relatively high when compared to the report of 4 in 10,000 in healthy children, however, this was similar to the previous reports of $3-26 \%$ in post-LT children. ${ }^{16-19}$

The significant culprit foods were cow's milk, hen's egg, shellfish, fish, wheat, and soy. Reactions to these "major allergens" are similar to those occurring in Thai children population. ${ }^{4,5}$ In addition, these are not different from previous reports of de novo $\mathrm{FA}$ or food sensitization in children after LT. ${ }^{9}$ Considering the common food allergens among Asian countries, cow's milk protein was the most common in our study 
while Shoda et al. reported that wheat was the most common in de novo FA in Japan. ${ }^{20}$ Our finding of multiple food allergies, occurring approximately $2 / 3(65.4 \%)$ of de novo FA patients, supported the consistence of published literature of de novo FA in pediatric LT. ${ }^{6,82,22}$ Furthermore, they lead to multiple food avoidance causing the huge negative impact on the nutritional achievement and quality of life post LT.

Although, the prevalence of food allergy was high in our LT patients, the accumulative outgrowing rate during 3 years after food elimination reached 19\%, particularly cow's milk, soy, wheat, and egg allergies. These findings were similar to childhood FA in normal population for cow's milk, hen's egg, soy, and wheat which typically resolves by 3 years of age. ${ }^{2}$ In addition, other studies have supported that de novo FA after LT is a transient condition and can be outgrown after food elimination for several years. ${ }^{21,23,24}$ Interestingly, our data presented a high rate of outgrowing of IgE-mediated shellfish allergy up to $2 / 3$ which was contrast to the natural history in normal population showing a low chance to develop tolerance. ${ }^{25}$

The pathogenesis of de novo FA is unknown. Potential mechanism related to chronic tacrolimus use and imbalance immunological function in liver themselves, have been hypothesized base on the reports in LT children both in retrospective, prospective, case-controlled, and in vitro studies. $^{6,9,11,12,26,27}$ Chronic exposure to oral tacrolimus, the calcineurin inhibitor, might skew the Th2 response and alter gastrointestinal barrier, and eventually increases a risk of FA development due to the improper allergen exposure. ${ }^{28}$ However, the present study showed that outgrowing of de novo FA patients did not related to a change in immunosuppressive protocol. Therefore, we speculate the potential etiologies of de novo FA are multifactorial rather than from long-term tacrolimus use only. More studies are required to identify the exact mechanism of de novo FA after LT.

Potential risk factors associated with de novo FA are controversial. Our study identified family history of atopy, young age at LT, particularly less than 2 years old, and EBV viremia as the risk factors. Several studies support our finding that LT at young age contributes to de novo FA. ${ }^{11,16,27,29}$ The potential mechanism of young age and risk for de novo FA probably due to the immature immune tolerance mechanism in gastrointestinal and hepatic themselves. ${ }^{2,9}$ Other reported potential risk factors included high PELD score prior to LT, underlying liver diseases, immunosuppressive regimens and donor's allergy. ${ }^{20,27,29}$ However, we could not demonstrate the association of these factors with de novo FA. The discrepancy of these results among studies could be due to a difference in study design, the definition of FA and outcome measurement of FA. Moreover, most of our LT patients were biliary atresia with the same level of severity of liver failure before LT and received the homogeneous immunosuppressive regimen.

EBV is a herpes virus with contagious and suspected to be associated with several allergic diseases in normal population. $^{30,31}$ Currently, the role of EBV infection in FA is controversial due to inconsistent results from both epidemiological and in vitro studies. It has been suspected to be either a risk or protective factor for FA..$^{30,32}$ Contributing factors for the variable results include the variety of EBV detection methods, the range of age group, co-infection with cytomegalovirus (CMV), and the different endpoint outcomes (sensitization or allergy). ${ }^{8,30,33}$ Most of the study evaluated the seroprevalence that may be inadequate to represent the viral activity. EBV, particularly primary infection, may related to atopic diseases due to it induces $\mathrm{B}$ cell proliferation and polyclonal antibody proliferation. ${ }^{31,32}$ In addition, it may transform human B cells, resulting in enhance interleukin-5 production, and eventually may induce a chronic eosinophilic inflammation and produce interleukin-4, which has an important role in promoting the production of the $\operatorname{IgE}$ antibodies. ${ }^{32,34}$ Therefore, we hypothesized that the EBV viremia which represents the reactivated EBV infection or viral replication, might exaggerate or precipitate the presentation of FA through activated IgE producing B cell proliferation and Th2 response. However, we did not examine the IgE producing B cell during EBV viremia in our patients to prove our hypothesis which beyond the scope of this retrospective study. Whether EBV viremia is the co-incidence finding or causal association with de novo FA requires further investigations.

Sidorchuk et al. reported the interaction of CMV and EBV infection on allergic diseases (asthma, allergic rhinitis, atopic dermatitis, but not include food allergy) and sensitization in the birth cohort survey. They found that the sensitization to common airborne or food allergens tended to be more prevalent in children with CMV-seropositive particularly when combined with EBV-seronegative. ${ }^{35}$ In contrast, the present study detected both EBV and CMV viremia in patients with de novo FA with the CMV reactivation rate of $68 \%$ but none in those without FA, however, there was insufficient statistical assessment due to inadequate sample of CMV viremia in the latter group. The large cohort studies are helpful to clarify this association in de novo FA post LT.

To our knowledge, this study possibly be the first report of de novo FA after LT in Southeast Asia that could be our strength. In addition, we provided data of long-term follow-up that was enough to discover the long-term outcome of these allergic diseases. FA was also diagnosed by the same allergists and gastroenterologists with the standard supportive evidence of sensitization and tissue biopsy. However, the study had some limitations due to the nature of retrospective research, therefore, recall bias could occur such as uncertainty of timing to develop or outgrowing of FA. The overestimation of the prevalence rate of de novo $\mathrm{FA}$ was also the concerned issue, nevertheless, we tried to diminish this issue by classifying the diagnosis of de novo FA to be confirmed and probable FA. The confirmed de novo FA was $39.1 \%$ which was still higher than normal children population. In addition, the patients who suspected de novo FA post LT had not designed to regularly re-challenge to evaluate the outgrown, thus, the precise timing of outgrown was difficult to clarify from this study.

In conclusion, de novo FA after LT in pediatric patients is not uncommon. It suggests that children after LT, carry a high risk for the development of new onset FA. Age at transplantation less than 2 years, family history of atopy, and EBV viremia associated with the developing FA. Nevertheless, the natural history of this de novo FA is similar to general pediatric population with food allergy with $19 \%$ of the patients develop tolerance after 3 years of food elimination. The diagnosis is 
challenging, therefore, high index of suspicion of FA should be considered in post-LT children. In addition, the larger cohort study should be further conducted to enhance the understanding of de novo FA in post-LT children.

\section{Acknowledgement}

There is no conflict of interest. We would like to acknowledge all liver transplant patients and their parents to participate in this study and Ms. Teerarat Tassanapitikul and Mr. Siam Saetang, Faculty of Medicine Ramathibodi Hospital for their assistance in data analysis. This study was supported by a research grant from the Faculty of Medicine Ramathibodi Hospital, Mahidol University.

\section{References}

1. Sampson HA, Aceves S, Bock SA, James J, Jones S, Lang D, et al. Food allergy: A practice parameter update-2014. J Allergy Clin Immunol. 2014; 1345:1016-25.e43.

2. Sicherer SH, Sampson HA. Food allergy. J Allergy Clin Immunol. 2010;125 (2 Suppl 2):S116-25.

3. Bock SA. Prospective appraisal of complaints of adverse reactions to foods in children during the first 3 years of life. Pediatrics. 1987;795:683-8.

4. Santadusit S, Atthapaisalsarudee S, Vichyanond P. Prevalence of adverse food reactions and food allergy among thai children. J Med Assoc Thai. 2005;88 Suppl 8:S27-32.

5. Lao-araya M, Trakultivakorn M. Prevalence of food allergy among preschool children in northern thailand. Pediatr Int. 2012;542:238-43.

6. Needham JM, Nicholas SK, Davis CM. Food allergies developing after solid organ transplant. Pediatr Transplant. 2015;198:827-35.

7. LaRosa C, Baluarte HJ, Meyers KE. Outcomes in pediatric solid-organ transplantation. Pediatr Transplant. 2011;152:128-41.

8. Ozbek OY, Ozcay F, Avci Z, Haberal A, Haberal M. Food allergy after liver transplantation in children: A prospective study. Pediatr Allergy Immunol. 2009;208:741-7.

9. Levy Y, Davidovits M, Cleper R, Shapiro R. New-onset post-transplantation food allergy in children-is it attributable only to the immunosuppressive protocol? Pediatr Transplant. 2009;131:63-9.

10. Noble C, Peake J, Lewindon PJ. Increase in de novo allergies after paediatric liver transplantation: The brisbane experience. Pediatr Transplant. 2011;155:451-4.

11. De Bruyne R, Dullaers M, Van Biervliet S, Vande Velde S, Raes A, Gevaert P, et al. Post-transplant food allergy in children is associated with liver and not with renal transplantation: A monocentric comparative study. Eur J Pediatr. 2013;1728:1069-75.

12. Boyle RJ, Hardikar W, Tang ML. The development of food allergy after liver transplantation. Liver Transpl. 2005;113:326-30.

13. Atkins D, Malka-Rais J. Food allergy: Transfused and transplanted. Curr Allergy Asthma Rep. 2010;104:250-7.

14. Burks AW, Tang M, Sicherer S, Muraro A, Eigenmann PA, Ebisawa M, et al. Icon: Food allergy. J Allergy Clin Immunol. 2012;1294:906-20.

15. Rothenberg ME. Eosinophilic gastrointestinal disorders (egid). J Allergy Clin Immunol. 2004;1131:11-28.

16. Wisniewski J, Lieberman J, Nowak-Wegrzyn A, Kerkar N, Arnon R, Iyer K, et al. De novo food sensitization and eosinophilic gastrointestinal disease in children post-liver transplantation. Clin Transplant. 2012;264:E365-71.
17. Parashette KR, Zeytinoglu M, Kernek K, Molleston JP, Subbarao G. Clinical, endoscopic, and histologic features of eosinophilic inflammation of the gastrointestinal tract in pediatric liver transplant patients. Pediatr Transplant. 2013;178:737-43.

18. Noble C, Francis L, Withers GW, Ee LC, Lewindon PJ. Audit of eosinophilic oesophagitis in children post-liver transplant. Pediatr Transplant. 2009;137:827-30

19. Uppal V, Kreiger P, Kutsch E. Eosinophilic gastroenteritis and colitis: A comprehensive review. Clin Rev Allergy Immunol. 2016;502:175-88.

20. Shoda T, Nomura I, Futamura M, Horimukai K, Narita M, Ohya Y, et al. The acquisition of food allergy in children after liver transplantation. J Allergy Clin Immunol. 2012;1292:AB35.

21. Mavroudi A, Xinias I, Deligiannidis A, Parapanissiou E, Imvrios G. Long term outcome of acquired food allergy in pediatric liver recipients: A single center experience. Pediatr Rep. 2012;41:e6.

22. Ozdemir O, Arrey-Mensah A, Sorensen RU. Development of multiple food allergies in children taking tacrolimus after heart and liver transplantation. Pediatr Transplant. 2006;103:380-3.

23. Frischmeyer-Guerrerio PA, Wisniewski J, Wood RA, Nowak-Wegrzyn A. Manifestations and long-term outcome of food allergy in children after solid organ transplantation. J Allergy Clin Immunol. 2008;1225:1031-3.e1.

24. Maarof G, Krzysiek R, Decline JL, Cohen J, Habes D, Jacquemin E. Management of post-liver transplant-associated ige-mediated food allergy in children. J Allergy Clin Immunol. 2011;1275:1296-8.

25. Lee AJ, Gerez I, Shek LP, Lee BW. Shellfish allergy--an asia-pacific perspective. Asian Pac J Allergy Immunol. 2012;301:3-10.

26. Manuyakorn W, Sinitkul R, Treepongkaruna S, Kamchaisatian W, Vilaiyuk $\mathrm{S}$, Srisala $\mathrm{S}$, et al. In vitro cytokine changes after pediatric liver transplantation. Asian Pac J Allergy Immunol. 2015;331:52-8.

27. Brown C, Haringman N, Davies C, Gore C, Hussain M, Mieli-Vergani G, et al. High prevalence of food sensitisation in young children with liver disease: A clue to food allergy pathogenesis? Pediatr Allergy Immunol. 2012;238:771-8.

28. Atkins FM. Systemic fk506 and post transplant food allergy in children. J Pediatr Gastroenterol Nutr. 2003;374:525-6.

29. Ozdemir O. New developments in transplant-acquired allergies. World J Transplant. 2013;33:30-5.

30. Sidorchuk A, Lagarde F, Pershagen G, Wickman M, Linde A. Epstein-barr virus infection is not associated with development of allergy in children. Pediatr Infect Dis J. 2003;227:642-7.

31. Okudaira H, Mori A. Concepts of the pathogenesis of allergic disease Possible roles of epstein-barr virus infection and interleukin-2 production. Int Arch Allergy Immunol. 1999;1203:177-84.

32. Pan Y, Nie Z, Zhang Y, Zhang K, Li J, Wang L. Identification of ebv infection in adults with egg specific food allergy. Virol J. 2013;10:9.

33. Catal F, Topal E, Selimoglu MA, Karabiber H, Baskiran A, Senbaba E, et al Acquired ige-mediated food allergy after liver transplantation in children. Allergol Immunopathol (Madr). 2015;434:392-7.

34. Nilsson C, Larsson Sigfrinius AK, Montgomery SM, Sverremark-Ekstrom E, Linde A, Lilja G, et al. Epstein-barr virus and cytomegalovirus are differentially associated with numbers of cytokine-producing cells and early atopy. Clin Exp Allergy. 2009;394:509-17.

35. Sidorchuk A, Wickman M, Pershagen G, Lagarde F, Linde A. Cytomegalovirus infection and development of allergic diseases in early childhood: Interaction with ebv infection? J Allergy Clin Immunol. 2004; 1146:1434-40. 